\title{
It is time to consider an anti-inflammatory therapy based on the pathophysiology of COVID-19 infection during the right time window?
}

Mostafa Ghanei

Chemical Injuries Research Centre, Systems Biology and Poisoning Institute, Baqiyatallah University of Medical Sciences, Tehran, Iran

Submitted: 24 October 2020; Accepted: 19 November 2020; Online publication: 26 February 2021

Arch Med Sci 2021; 17 (2): 546-550

DOI: https://doi.org/10.5114/aoms/130647

Copyright $\odot 2021$ Termedia \& Banach

The main chest computed tomography (CT) imaging features of COVID-19 have been previously described [1-4]. Based on chest CT scans (e.g. the reverse halo sign), linear consolidations, and other signs indicate an organising pneumonia pattern [3]. Pathological characteristics such as pulmonary oedema and hyaline membrane formation are of great importance for timely and appropriate administration of corticosteroids in critically ill COVID-19 patients. Pathological evidence has revealed the pulmonary tissue pathology, where an acute fibrinous (in the initial stage) and organising pneumonia (AFOP) can be described based on the intra-alveolar fibrin deposits in some critically ill COVID patients. This indicates a cortico-sensitive pathology [5]. Autopsy series have further indicated that the predominant histological pattern in severe COVID-19 patients is organising pneumonia (OP), not diffuse alveolar damage (DAD) [6]. Thus, an insight into the empirical corticosteroid therapy of COVID-19 might be reconsidered based on the histological pattern of lung injury and cytokine storm-associated hyperinflammation. Despite multiple targeted therapeutic approaches and/or use of anti-viral agents, no specific effective therapeutic strategy is available for COVID-19. On the other hand, various clinical trials of potential therapies as well as vaccines are ongoing. While the hyperinflammatory phase with cytokine storm is linked to the histological pattern of severe COVID-19 [4], patchy inflammatory cell infiltration has also been demonstrated at the early stage of COVID-19 pathology [7].

COVID-19 therefore shows 2 classic phases of the immune response: a protective immune defence against the virus and inflammation-driven tissue/organ damage. Hence, immune-boosting strategies such as potentiation of serum immunity or pegylated IFN- $\alpha$ and antiviral strategies may be suggested in the early or non-severe stage of COVID-19. On the other hand, immunosuppression/immunomodulatory strategies (e.g. corticosteroids, immunomodulators, inflammatory cytokine antagonists such as tocilizumab [8] or anakinra, decrease of lung inflammatory cell infiltration, etc.) in the inflammation-driven damaging phases can be considered [9]. Therefore, anti-inflammatory approach at the wrong time (in the early phase) may be potentially linked to deleterious effects, or may not even be beneficial for consolidated and irreversible tissue damage.

This indicates that the cytokine storm condition in COVID-19, which is associated with the severe pathology, can be targeted by the anti-inflammatory strategy. Virus-induced immunopathological events have been

\author{
Corresponding author: \\ Mostafa Ghanei \\ Chemical Injuries Research \\ Centre \\ Systems Biology \\ and Poisoning Institute \\ Baqiyatallah University \\ of Medical Sciences \\ Tehran, Iran \\ E-mail: \\ mghaneister@gmail.com
}


shown to be associated with COVID-19 pneumonia, suggesting a timely tailored therapy based on the pathophysiology of COVID-19. Additionally, AFOP pattern reveals an open question regarding the use of corticosteroid therapy.

While there is no doubt that antiviral strategy has a special place in fighting COVID-19, an antiinflammatory regimen (e.g. corticosteroids, etc.) may be hypothetically considered as a tailored combination therapy in preventing further injury and improving the treatment success rate among COVID-19 patients during this extremely short time window.

Short-term administration of low-dose corticosteroids may show beneficial effects in the treatment of COVID-19 patients, especially severe patients, by considering their wise and timely use as well as gradual tapering [10-15]. Based on the sensitivity of the AFOP pattern to glucocorticoids, however, questions about the uncertain risks, type of pulmonary lesion, and the appropriate timing remain to be answered in terms of COVID-19. Compelling evidence so far suggests the benefit of steroids (methylprednisolone, and dexamethasone in patients with severe COVID-19) [16, 17]. The application of corticosteroids has been suggested in the multinational Surviving Sepsis Guidelines for patients requiring mechanical ventilation [18]. Based on this, the WHO has updated its guidelines to suggest steroid use (e.g. dexamethasone) for severe COVID-19 associated with hypoxemic respiratory failure [19]. Reliable evidence on the beneficial effects of immunomodulating drugs such as anti-tumour necrosis factor (TNF) drugs anti-IL6, and antiIL-1 are scarce in the clinical setting and require further in-depth investigations in clinical trials.

In the early stages of the COVID-19 pandemic it was reported that azithromycin alone as a macrolide or in combination with hydroxychloroquine may be beneficially effective in the management of COVID-19 patients with a potential safety signal [20,21], because of its immunomodulatory and postulated antiviral properties $[22,23]$. Additionally, the efficacy of azithromycin for improving the phagocytic function of macrophages has been described in chronic obstructive pulmonary disease (COPD) [24].

With immunomodulatory properties, macrolides mitigate anti-inflammatory cytokines and increase the levels of immunoglobulins [25]. Preclinical and clinical findings on macrolides in respiratory infections as well as preliminary evidence from COVID-19 signifies the potential beneficial effects of azithromycin in combating COVID-19 [26-28]. An $\mathrm{EC}_{50}$ of $2.12 \mu \mathrm{M}$ against COVID-19 has been proven for azithromycin in in vitro screening [27].

A limited number of clinical studies suggested that administration of hydroxychloroquine may enhance the early clinical recovery of COVID-19 pa- tients. The efficacy of hydroxychloroquine has been demonstrated for 62 moderately ill patients [29]. In contrast, the findings of a RECOVERY trial by Horby et al. revealed findings against the beneficial effect of hydroxychloroquine administration on a population of 4674 COVID-19 patients [30].

In a French clinical trial, the high efficacy of the combination of hydroxychloroquine with azithromycin has been reported as 100\% among COVID-19 patients virologically cured at day 6 , whereas $57.1 \%$ of patients benefited from only hydroxychloroquine [31]. In contrast, no beneficial effect or rapid viral clearance of this combination have been found at the same dose scheme for the severe COVID-19 in an uncontrolled observational study [32]. Additionally, no beneficial effects of this combination or hydroxychloroquine alone have been found for either postexposure prophylaxis or COVID-19 under randomised, controlled trials [33-35], while in-hospital mortality was not found to be different among COVID-19 patients receiving hydroxychloroquine, azithromycin, or their combination, as compared to patients receiving no treatments [36]. On the other hand, other investigations indicated the need for caution, especially regarding patients with underlying arrhythmia due to QT prolongation or torsades de pointes (TdP) $[21,37]$. In a systematic review, early administration of hydroxychloroquine has been indicated to be effective in the outpatient setting for COVID-19 [38], while no beneficial effect has been revealed for the use of hydroxychloroquine as a readily available drug in patients with mild to moderate COVID-19 [39]. The WHO discontinued the therapeutic arms of the Solidarity trial on 4 July 2020 based on the results because of a lack of beneficial effects, which was in agreement with the findings from the RECOVERY trial of COVID-19 [40].

However, ongoing trials on hydroxychloroquine can answer the controversy because hydroxychloroquine was not linked to a lower death at 28 days among hospitalised patients with COVID-19 when compared to those who received the usual treatment, as reported by the RECOVERY Collaborative Group [41].

Non-steroidal anti-inflammatory drugs (NSAIDs) are capable of disrupting the resolution of the inflammatory process. In this regard, naproxen may be considered for patients suffering from severe COVID-19 in inflammation-driven damaging phases, due to its triple effects including anti-inflammatory, anti-influenza virus [42], and antiplatelet properties. No conclusive evidence so far suggests its risk for severe adverse events (e.g. death) in patients with COVID-19 [43], while a cautionary approach is suggested for patients with non-severe symptoms [44]. Nonetheless, pending further investigation, its benefits and risks will be established in the near future. 
Increasing evidence demonstrates the association of COVID-19 with coagulopathy and thrombosis risk, especially in severely ill patients [45-48], where providing the role of D-dimer or scoring systems is of great importance for stratifying patients' risk.

Emerging evidence suggests that statins play a positive role in the improvement of outcomes among COVID-19 patients (e.g. reduction of the risk of mortality and its severity) due to their pleiotropic features (e.g. anti-inflammatory and anti-thrombotic features, etc.). Therefore, de novo use of statins has been suggested for COVID-19 patients without underlying comorbidities [49, 50], but determination of the benefit/risk of statins requires further studies under clinical conditions [51].

Although corticosteroids worsen the glycaemic control in diabetic patients, their use may be considered after balancing glucose levels by using glucose-lowering therapies. The corticosteroid therapy for diabetic patients with COVID-19 requires consideration of the probability of uncontrolled glucose levels. However, management of diabetic patients is challenging for decreasing the risk of complications and death. On the other hand, certain inflammatory markers in COVID-19 patients might be linked to poor glycaemic control; thus, euglycaemia is important in diabetic patients during COVID-19 circumstance [52].

Thrombotic event monitoring for COVID-19 inpatients is of great importance, and patients with bleeding episodes should be managed.

Antithrombotic therapy (antiplatelet or anticoagulant administration) for preventive, prophylaxis, and treatment approaches can also be recommended based on the clinical manifestation and the severity of COVID-19 in patients with signs of coagulopathy. There are rationales behind antiplatelet, corticosteroid, and anti-cytokine treatments to stabilise the endothelium and platelets [53].

COVID-19 disease is a challenging issue for patients suffering from inflammatory autoimmune systemic diseases (ASD), and a study by Ferri et al. indicated a higher occurrence of COVID-19 infection among patients with ASD in Italy, indicating the need to provide preventive/management strategies [54]. Susceptibility of individuals with rheumatic disease to COVID-19 has been reported by an observational study in Hubei province, China [55]. Conversely, another study indicated that autoimmune rheumatic disease was not related to a considerable risk of COVID-19 disease or its severity among adults and paediatrics [56]; such findings indicate the need for clarification of the susceptibility of patients with ASD to COVID-19 and the possible interactions between COVID-19 and ASD. Therefore, the possibility of the risks/ benefits of anti-rheumatic therapy needs clari- fication, but the use of low-dose corticosteroids and bDMARDs has been recommended at various times for COVID-19 patients [57].

Given the lack of specific antivirals for COVID-19, a tailored and accessible anti-inflammatory treatment regimen including azithromycin, prednisolone, naproxen, and statins may be envisaged. This is to target the inflammation-driven damaging phases of COVID-19 pathology through appropriate assessment of the risk/benefit ratio. Furthermore, the use of antivirals with high lung distribution profiles may be an effective strategy to decrease viral loads of SARS-CoV-2 [58].

Lopinavir/ritonavir and remdesivir are among the drugs with poor lung distributions, and thus are considered to be inadequate for the inhibition of SARS-CoV-2 $[59,60]$.

However, well-designed randomised clinical trials are of great importance to pave the way for assessing the risks/benefits of such drugs. We conclude that in our opinion the use of expensive antiviral drugs, often with unproved efficacy, may deprive a large population of COVID-19 patients in the world (particularly in less developed countries) from appropriate treatment. On the other hand, an anti-inflammatory approach using widely accessible drugs could be effective if applied during the right time window of COVID-19 pathology, and hence we recommend this approach.

\section{Conflict of interest}

The authors declare no conflict of interest

\section{References}

1. Shi $\mathrm{H}$, Han X, Jiang N, et al. Radiological finding from 81 patients with COVID-19 pneumonia in Wuhan, China: a descriptive study. Lancet Infect Dis 2020; 20: 425-34.

2. Bhaskar S, Sinha A, Banach M, et al. Cytokine storm in COVID-19 - immunopathological mechanisms, clinical considerations, and therapeutic approaches: the REPROGRAM Consortium position paper. Front Immunol 2020; 11: 1648

3. Hani C, Trieu NH, Saab I, et al. COVID-19 pneumonia: a review of typical CT findings and differential diagnosis. Diagn Interv Imaging 2020; 1: 263-8.

4. Xu Z, Shi L, Wang Y, et al. Pathological findings of COVID-19 associated with acute respiratory distress syndrome. Lancet Respir Med 2020; 8: 420-2.

5. Copin MC, Parmentier E, Duburcq T, Poissy J, Mathieu D. Time to consider histologic pattern of lung injury to treat critically ill patients with COVID-19 infection. Intensive Care Med 2020; 23: 1-3.

6. Flikweert AW, Grootenboers MJ., Yick DC, et al. Late histopathologic characteristics of critically ill COVID-19 patients: different phenotypes without evidence of invasive aspergillosis, a case series. J Crit Care 2020; 59: 149-55.

7. Tian SH, Hu W, Niu L, Liu H, Xu H, Xiao S. Pulmonary pathology of early phase SARS-COV-2 pneumonia. J Thorac Oncol 2020; 15: 700-4. 
8. Liberato NL, De Monte A, Caravella G. Tocilizumab in severe COVID-19. Arch Med Sci 2020; 16: 1457-8.

9. Shi Y, Wang Y, Shao C, et al. COVID-19 infection: the perspectives on immune responses. Cell Death Differ 2020; 27: 1451-4.

10. Wu C, Chen X, Cai Y, et al. Risk factors associated with acute respiratory distress syndrome and death in patients with coronavirus disease 2019 pneumonia in Wuhan, China. JAMA Intern Med 2020; 180: 1-11.

11. Zheng C, Wang J, Guo H, et al. Risk-adapted treatment strategy for COVID-19 patients. Int J Infect Dis 2020; 94: 74-77.

12. Zhou ZG, Xie SM, Zhang J, et al. Short-term moderate-dose corticosteroid plus immunoglobulin effectively reverses COVID-19 patients who have failed low-dose therapy. Preprints (www.preprints.org) 2020. DOI: 10.20944/preprints202003.0065.v1.

13. Shang L, Zhao J, Hu Y, Du R, Cao B. On the use of corticosteroids for 2019-nCoV pneumonia. Lancet 2020; 395: 683-4.

14. Goursaud S, Descamps R, Daubin C, du Cheyron D, Valette X. Corticosteroid use in selected patients with severe acute respiratory distress syndrome related to Covid-19. J Infect 2020; 81: e89-90.

15. Taboada M, Caruezo V, Naveira A, Atanassoff PG. Corticosteroids and the hyper-inflammatory phase of the COVID-19 disease. J Clin Anesth 2020; 66: 109926.

16. RECOVERY Collaborative Group. Dexamethasone in hospitalized patients with Covid-19 - preliminary report. N Engl J Med 2020. DOI: 10.1056/NEJMoa2021436.

17. Wang $\mathrm{Y}$, Jiang $\mathrm{W}, \mathrm{He} \mathrm{Q}$, et al. A retrospective cohort study of methylprednisolone therapy in severe patients with COVID-19 pneumonia Sig. Transduct Target Ther 2020; 5: 57.

18. Alhazzani W, Moller M, Arabi YM, et al. Surviving Sepsis Campaign: guidelines on the management of critically ill adults with Coronavirus Disease 2019 (COVID-19). Intensive Care Med 2020; 46: 854-87.

19. WHO. Dexamethasone and COVID-19; 25 June 2020; https://www.who.int/news-room/q-a-detail/q-a-dexamethasone-and-covid-19.

20. Sarayani A, Cicali B, Henriksen CH, Brown JD. Safety signals for QT prolongation or Torsades de Pointes associated with azithromycin with or without chloroquine or hydroxychloroquine. Res Social Adm Pharm 2020. DOI: 10.1016/j.sapharm.2020.04.016.

21. Lane JCE, Weaver J, Kostka K, et al. Safety of hydroxychloroquine, alone and in combination with azithromycin, in light of rapid wide-spread use for COVID-19: a multinational, network cohort and self-controlled case series study. medRxiv 2020. 2020.2004.2008.20054551

22. Parnham MJ, Haber VE, Giamarellos-Bourboulis EJ, Perletti G, Verleden GM, Vos R. Azithromycin: mechanisms of action and their relevance for clinical applications. Pharmacol Ther 2014; 143: 225-45.

23. Madrid PB, Panchal RG, Warren TK, et al. Evaluation of Ebola virus inhibitors for drug repurposing. ACS Infect Dis 2016; 1: 317-26.

24. Hodge S, Hodge G, Brozyna S, Jersmann H, Holmes M, Reynolds PN. Azithromycin increases phagocytosis of apoptotic bronchial epithelial cells by alveolar macrophages. Eur Respir I 2006; 28: 486-95.

25. Bermejo-Martin JF, Kelvin DJ, Eiros JM, Castrodeza J, Ortiz de Lejarazu R. Macrolides for the treatment of severe respiratory illness caused by novel H1N1 swine influenza viral strains. J Infect Dev Ctries 2009; 3: 159-61.
26. Arabi YM, Deeb AM, Al-Hameed F, et al. Macrolides in critically ill patients with middle east respiratory syndrome. Int J Infect Dis 2019; 81: 184-90.

27. Touret F, Gilles $M$, Barral K, et al. In vitro screening of a FDA approved chemical library reveals potential inhibitors of SARS-CoV-2 replication. Sci Rep 20; 10: 13093.

28. Pani A, Lauriola M, Romandini A, Scaglione F. Macrolides and viral infections: focus on azithromycin in COVID-19 pathology. Int J Antimicrob Agents 2020; 10: 106053.

29. Chen Z, Hu J, Zhang Z, et al. Efficacy of hydroxychloroquine in patients with COVID-19: results of a randomized clinical trial. MedRxiv 2020. DOI: https://doi.org/10 $.1101 / 2020.03 .22 .20040758$

30. Horby P, Landray M. No clinical benefit from use of hydroxychloroquine in hospitalised patients with COVID-19. Available from: http://www.ox.ac.uk/news/ 2020-06-05-no-clinical-benefit-use-hydroxychloroquine-hospitalised-patients-covid-19.

31. Gautret P, Lagier JC, Parola P, et al. Hydroxychloroquine and azithromycin as a treatment of COVID-19: results of an open-label non-randomized clinical trial. Int J Antimicrob Agents 2020; 56: 105949.

32. Molina JM, Delaugerre C, Le Goff J, Mela-Lima B, Ponscarme D, De Castro N. No evidence of rapid antiviral clearance or clinical benefit with the combination of hydroxychloroquine and azithromycin in patients with severe COVID-19 infection. Med Mal Infect 2020; 50: 384.

33. Tang W, Cao Z, Han M, et al. Hydroxychloroquine in patients with mainly mild to moderate coronavirus disease 2019: open label, randomised controlled trial. BMJ 2020; 369: m1849.

34. Boulware DR, Pullen MF, Bangdiwala AS, et al. A randomized trial of hydroxychloroquine as postexposure prophylaxis for Covid-19. N Engl J Med 2020; 383: 517-25.

35. Cavalcanti AB, Zampieri FG, Rosa RG, et al. Hydroxychloroquine with or without azithromycin in mild-to-moderate Covid-19. N Engl J Med 2020; 383: e119.

36. Rosenberg ES, Dufort EM, Udo T, et al. Association of treatment with hydroxychloroquine or azithromycin with in-hospital mortality in patients with COVID-19 in New York state. JAMA 2020; 323: 2493-502.

37. Nguyen LS, Dolladille C, Drici MD, et al. Cardiovascular toxicities associated with hydroxychloroquine and azithromycin: an analysis of the World Health Organization pharmacovigilance Database. Circulation 2020; 142: 303-5.

38. Prodromos C, Rumschlag T. Hydroxychloroquine is effective, and consistently so used early, for Covid-19: a systematic review. New Microbes New Infect 2020; 38: 100776.

39. Pathak SK, Salunke AA, Thivari P, et al. No benefit of hydroxychloroquine in COVID-19: results of systematic review and meta-analysis of randomized controlled trials. Diabetes Metab Syndr 2020; 14: 1673-80.

40. World Health Organization. WHO discontinues hydroxychloroquine and lopinavir/ritonavir treatment arms for COVID-19. July 4, 2020. Available at: https://www. who.int/news-room/detail/04-07-2020-who-discontinues-hydroxychloroquine-and-lopinavir-ritonavir-treatment-arms-for-covid-19.

41. RECOVERY Collaborative Group; Horby P, Mafham M, Linsell L, et al. Effect of hydroxychloroquine in hospitalized patients with Covid-19. N Engl J Med 2020; 383: 2030-40.

42. Lejal N, Tarus B, Bouguyon E, et al. Structure-based discovery of the novel antiviral properties of naproxen 
against the nucleoprotein of influenza a virus. Antimicrob Agents Chemother 2013; 57: 2231-42.

43. Micallef J, Soeiro T, Annie-Pierre JB. Non-steroidal antiinflammatory drugs, pharmacology, and COVID-19 infection. Therapies 2020; 75: 355-62.

44. NICE. COVID-19 rapid guideline: managing symptoms (including at the end of life) in the community (2020). Available at: https://www.nice.org.uk/guidance/NG163 [Accessed May 4, 2020]

45. Danzi GB, Loffi M, Galeazzi G, Gherbesi E. Acute pulmonary embolism and COVID-19 pneumonia: a random association? Eur Heart J 2020; 41: 1858.

46. Tang N, Li D, Wang X, Sun Z. Abnormal coagulation parameters are associated with poor prognosis in patients with novel coronavirus pneumonia. J Thromb Haemost 2020; 18: 844-7.

47. Kollias A, Kyriakoulis KG, Dimakakos E, Poulakou G, Stergiou GS, Syrigos K. Thromboembolic risk and anticoagulant therapy in COVID-19 patients: emerging evidence and call for action. Br J Haematol 2020; 189: 846-7.

48. Al-Ani F, Chehade S, Lazo-Langner A. Thrombosis risk associated with COVID-19 infection. A scoping review. Thromb Res 2020; 192: 152-60.

49. Subir R. Pros and cons for use of statins in people with coronavirus disease-19 (COVID-19). Diabetes \& Metabolic Syndrome: Diabetes Metab Syndr 2020; 14: 1225-9.

50. Katsiki N, Banach M, Mikhailidis DP. Lipid-lowering therapy and renin-angiotensin-aldosterone system inhibitors in the era of the COVID-19 pandemic. Arch Med Sci 2020; 16: 485-9.

51. Reiner Ž, Hatamipour M, Banach $M$, et al. Statins and the COVID-19 main protease: in silico evidence on direct interaction. Arch Med Sci 2020; 16: 490.

52. Apicella M, Campopiano MC, Mantuano M, Mazoni L, Coppelli A, Del Prato S. COVID-19 in people with diabetes: understanding the reasons for worse outcomes. Lancet Diabetes Endocrinol 2020; 8: 782-92.

53. Godino C, Scotti A, Maugeri N, et al. Antithrombotic therapy in patients with COVID-19? - rationale and evidence. Int J Cardiol 2021; 324: 261-6.

54. Ferri C, Giuggioli D, Raimondo V, et al. COVID-19 and rheumatic autoimmune systemic diseases: report of a large Italian patients series. Clin Rheumatol 2020; 39: 3195-204.

55. Zhong J, Shen G, Yang H, et al. COVID-19 in patients with rheumatic disease in Hubei province, China: a multicentre retrospective observational study. Lancet Rheumatol 2020; 2: e557-64.

56. Michelena X, Borrell H, López-Corbeto M, et al. Incidence of COVID-19 in a cohort of adult and paediatric patients with rheumatic diseases treated with targeted biologic and synthetic disease-modifying anti-rheumatic drugs. Semin Arthritis Rheum 2020; 50: 564-70.

57. Sarzi-Puttini P, Marotto D, Caporali R, et al. Prevalence of COVID infections in a population of rheumatic patients from Lombardy and Marche treated with biological drugs or small molecules: a multicentre retrospective study. J Autoimmun 2021; 116: 102545.

58. Sun D. Remdesivir for treatment of COVID-19: combination of pulmonary and IV administration may offer aditional benefit. AAPS J 2020; 26: 77

59. Wang Y, Chen L. Tissue distributions of antiviral drugs affect their capabilities of reducing viral loads in COVID-19 treatment. Eur J Pharmacol 2020; 889: 173634.

60. Liu C, Huang Q, Wang P, et al. COVID-19 disease: novel clinical manifestations and therapeutic exploration. Arch Med Sci 2020; 16: 485-9. 